PDES, SUBMANIFOLDS AND

AFFINE DIFFERENTIAL GEOMETRY

BANACH CENTER PUBLICATIONS, VOLUME 69

INSTITUTE OF MATHEMATICS

POLISH ACADEMY OF SCIENCES

WARSZAWA 2005

\title{
THE SPECTRAL GEOMETRY OF THE WEYL CONFORMAL TENSOR
}

\author{
N. BLAŽIĆ \\ Faculty of Mathematics, University of Belgrade \\ Studentski Trg 16, p.p. 550, 11000 Beograd, Serbia and Montenegro \\ E-mail: blazicn@matf.bg.ac.yu \\ P. GILKEY \\ Mathematics Department, University of Oregon, Eugene, OR 97403, U.S.A. \\ E-mail: gilkey@darkwing.uoregon.edu \\ S. NIKČEVIĆ \\ Mathematical Institute, SANU \\ Knez Mihailova 35, p.p. 367, 11001 Beograd, Serbia and Montenegro \\ E-mail: stanan@mi.sanu.ac.yu \\ U. SIMON \\ Fachbereich Mathematik, Technische Universität Berlin \\ Strasse des 17. Juni 135, D-10623 Berlin, Germany \\ E-mail:simon@math.tu-berlin.de
}

\begin{abstract}
We study when the Jacobi operator associated to the Weyl conformal curvature tensor has constant eigenvalues on the bundle of unit spacelike or timelike tangent vectors. This leads to questions in the conformal geometry of pseudo-Riemannian manifolds which generalize the Osserman conjecture to this setting. We also study similar questions related to the skewsymmetric curvature operator defined by the Weyl conformal curvature tensor.
\end{abstract}

\section{Introduction}

1.1. Algebraic curvature tensors. We work in a purely algebraic context for the moment. Consider a triple $\mathcal{V}:=(V, g, A)$ where $g$ is a non-degenerate inner product of signature

2000 Mathematics Subject Classification: Primary 53B20.

Key words and phrases: conformally flat, Ivanov-Petrova manifold, Jacobi operator, Osserman manifold, skew-symmetric curvature operator, Weyl conformal tensor.

The paper is in final form and no version of it will be published elsewhere. 
$(p, q)$ on a finite dimensional real vector space $V$ of dimension $m:=p+q \geq 3$ and where $A \in \otimes^{4} V^{*}$ is an algebraic curvature tensor on $V$; i.e. $A$ has the usual symmetries of the Riemann curvature tensor:

$$
\begin{aligned}
& A(x, y, z, w)=A(z, w, x, y)=-A(y, x, z, w), \quad \text { and } \\
& A(x, y, z, w)+A(y, z, x, w)+A(z, x, y, w)=0 .
\end{aligned}
$$

We say that $\mathcal{V}$ is Riemannian if $p=0$ and Lorentzian if $p=1$.

If $\phi$ is a $g$ self-adjoint endomorphism of $V$, then we set

$$
A_{\phi}(x, y, z, w):=g(\phi x, w) g(\phi y, z)-g(\phi x, z) g(\phi y, w) .
$$

Fiedler [4] showed these algebraic curvature tensors span the space $\mathcal{A}(V)$ of all algebraic curvature tensors. These tensors will play a crucial role in our subsequent discussion; setting $\phi=$ Id yields the algebraic curvature tensor of constant sectional curvature +1 .

1.2. The Weyl conformal curvature tensor. There is a natural representation of the orthogonal group $O(V, g)$ on $\mathcal{A}(V)$ defined by pull-back; if $A \in \mathcal{A}(V)$ and $\psi \in O(V, g)$, the pull-back $\psi^{*} A \in \mathcal{A}(V)$ is given by

$$
\left(\psi^{*} A\right)(x, y, z, w)=A(\psi x, \psi y, \psi z, \psi w) .
$$

This representation is not irreducible but decomposes as the direct sum of 3 irreducible representations which we can describe as follows. Let $g_{i j}:=g\left(e_{i}, e_{j}\right)$ and let $g^{i j}$ be the inverse matrix relative to some basis $\left\{e_{i}\right\}$ for $V$. The associated Ricci tensor $\rho_{A}$ and scalar curvature $\tau_{A}$ are then defined by contracting indices:

$$
\rho_{A}(x, y):=\sum_{i j} g^{i j} A\left(x, e_{i}, e_{j}, y\right) \quad \text { and } \quad \tau_{A}:=\sum_{i j} g^{i j} \rho_{A}\left(e_{i}, e_{j}\right)
$$

The associated maps $\sigma_{\rho}: A \rightarrow \rho_{A} \in S^{2}\left(V^{*}\right)$ and $\sigma_{\tau}: A \rightarrow \tau_{A} \in \mathbb{R}$ are $O(V, g)$ equivariant. The space $\mathcal{W}(V, g):=\operatorname{ker}\left(\sigma_{\rho}\right)$ of algebraic Weyl tensors is an irreducible representation space for $O(V, g)$ and we have:

$$
\mathcal{A}(V)=\mathcal{W}(V, g) \oplus S^{2}\left(V^{*}\right)
$$

as an $O(V, g)$ representation space. The further decomposition of $S^{2}\left(V^{*}\right)$ as the direct sum of the trace free tensors and the scalar multiples of the identity then completes the decomposition of $\mathcal{A}(V)$ as a direct sum of irreducible $O(V, g)$ modules. Let $\pi_{\mathcal{W}}$ be orthogonal projection from $\otimes{ }^{4} V^{*}$ to $\mathcal{W}(V, g)$ :

$$
\begin{aligned}
\pi_{\mathcal{W}}(A)(x, y, z, w)= & A(x, y, z, w)-\frac{1}{m-2}\left\{\rho_{A}(x, w) g(y, z)+g(x, w) \rho_{A}(y, z)\right\} \\
& +\frac{1}{m-2}\left\{\rho_{A}(x, z) g(y, w)+g(x, z) \rho_{A}(y, w)\right\} \\
& +\frac{1}{(m-1)(m-2)} \tau_{A}\{g(x, w) g(y, z)-g(x, z) g(y, w)\} .
\end{aligned}
$$

1.3. The Jacobi operator. If $A$ is an algebraic curvature tensor, then the Jacobi operator $J_{A}$ is a $g$ self-adjoint map of $V$ characterized by the property:

$$
g\left(J_{A}(x) y, z\right)=A(y, x, x, z) .
$$


For example, if $A=A_{\phi}$ is given by Equation (1.a), then

$$
J_{A_{\phi}}(x) y=g(\phi x, x) \phi y-g(\phi x, y) \phi x .
$$

It is clear that $\rho_{A}(x, x)=\operatorname{Tr}\left\{J_{A}(x)\right\}$ for any $A$; in particular

$$
\operatorname{Tr}\left\{J_{W}(x)\right\}=0 \quad \text { for any } \quad x \in V \quad \text { if } \quad W \in \mathcal{W}(V, g) .
$$

The pseudo-spheres of unit spacelike $(+)$ and unit timelike $(-)$ vectors in $V$ are

$$
S^{ \pm}(\mathcal{V}):=\{v \in V: g(v, v)= \pm 1\}
$$

We say that $\mathcal{V}$ is spacelike (resp. timelike) Jordan Osserman if the Jordan normal form of $J_{A}$ is constant on $S^{+}(\mathcal{V})$ (resp. on $S^{-}(\mathcal{V})$ ). If $\mathcal{V}$ is Riemannian, then the Jordan normal form is determined by the eigenvalue structure and, as every non-zero vector is spacelike, we shall drop the qualifiers 'spacelike' and 'Jordan' in the interests of notational simplicity. Note that the eigenvalue structure does not determine the Jordan normal form in the higher signature context.

1.4. The skew-symmetric curvature operator. Let $\left\{e_{1}, e_{2}\right\}$ be an orthonormal basis for an oriented spacelike (resp. timelike) 2 plane $\pi$ of $V$. One then defines the skew-symmetric curvature operator $A(\pi)$ by the identity:

$$
g(A(\pi) x, y):=A\left(e_{1}, e_{2}, x, y\right) .
$$

This $g$ skew-symmetric endomorphism of $V$ is independent of the particular oriented orthonormal basis for $\pi$ which is chosen. One says $\mathcal{V}$ is spacelike (resp. timelike) Jordan Ivanov-Petrova if the Jordan normal form of $A(\pi)$ is constant on the Grassmannian of oriented spacelike (resp. timelike) 2 planes in $V$.

1.5. The geometric setting. Let $R$ be the Riemann curvature tensor of a pseudo-Riemannian manifold $(M, g)$ of signature $(p, q)$ and dimension $m:=p+q \geq 3$. Let $\mathcal{R}_{P}:=$ $\left(T_{P} M, g_{P}, R_{P}\right)$ be the triple determined by the tangent bundle of $M$ at a point $P$ of $M$, the pseudo-Riemannian metric $g_{P}$, and the curvature tensor $R_{P}$.

We say that $(M, g)$ is pointwise spacelike (resp. timelike) Jordan Osserman if $\mathcal{R}_{P}$ is spacelike (resp. timelike) Jordan Osserman for every point $P$ of $M$; the Jordan normal form of $J_{R}$ is allowed to vary with the point $P$ of $M$. We say $(M, g)$ is globally spacelike (resp. timelike) Jordan Osserman if the Jordan normal form of $J_{R}$ on the appropriate pseudo-sphere bundle is independent of $P$. It is known that any global Riemannian $(p=0)$ Osserman manifold is locally isometric to a rank 1 symmetric space if $m \neq 16[3,18$, $20,19]$ and that any local Lorentzian $(p=1)$ Jordan Osserman manifold has constant sectional curvature $[1,5]$. In the higher signature setting, there exist spacelike and timelike Jordan Osserman manifolds which are not locally homogeneous [2, 7]. There is a vast literature on the subject and we shall content ourselves by refering to [6] for further details.

We say that $(M, g)$ is pointwise spacelike (resp. timelike) Jordan Ivanov-Petrova if $\mathcal{R}_{P}$ is spacelike (resp. timelike) Jordan Ivanov-Petrova for every point $P$ of $M$; again, the Jordan normal form of $J_{R}$ is allowed to vary with the point $P$ of $M$. These manifolds have been classified in the Riemannian setting if $m \neq 3,7[8,11,17]$, in the Lorentzian setting if $m \geq 11$ and if $\{m, m+1\}$ are not powers of $2[15,16]$, and in the higher signature 
setting if $q \geq 11$, if $p \leq \frac{q-6}{4}$, if $\{q, q+1, \ldots, q+p\}$ does not contain a power of 2 , and if $R(\pi)$ is not nilpotent [21]. We refer to [9] for further details concerning spacelike and timelike Jordan Ivanov-Petrova manifolds.

1.6. Conformal geometry. Let $P$ be a point of a pseudo-Riemannian manifold $(M, g)$. Let $\mathcal{W}_{P}:=\left(T_{P} M, g_{P}, W_{P}\right)$ where $W_{P}:=\pi_{\mathcal{W}} R_{P}$ is the associated Weyl conformal curvature tensor on $T_{P} M$. We say that $(M, g)$ is conformally spacelike (resp. timelike) Jordan Osserman if $\mathcal{W}_{P}$ is spacelike (resp. timelike) Jordan Osserman for every point $P$ of $M$. Similarly, we say that $(M, g)$ is conformally spacelike (resp. timelike) Jordan Ivanov-Petrova if $\mathcal{W}_{P}$ is spacelike (resp. timelike) Jordan Ivanov-Petrova for every point $P$ of $M$. In both settings, the Jordan normal form is permitted to vary with the point $P$ of $M$; the technical distinction between 'global' and 'pointwise' plays no role in this setting.

Recall that two metrics $g_{1}$ and $g_{2}$ are said to be conformally equivalent if there is a positive scaling function $\alpha \in C^{\infty}(M)$ so that $g_{1}=\alpha g_{2}$. We let $[g]$ be the set of all pseudo-Riemannian metrics on $M$ which are conformally equivalent to $g$.

THEOREM 1.1. Let $g_{1} \in\left[g_{2}\right]$. Then:

1. $\left(M, g_{1}\right)$ is conformally spacelike (resp. timelike) Jordan Osserman if and only if $\left(M, g_{2}\right)$ is conformally spacelike (resp. timelike) Jordan Osserman.

2. $\left(M, g_{1}\right)$ is conformally spacelike (resp. timelike) Jordan Ivanov-Petrova if and only if $\left(M, g_{2}\right)$ is conformally spacelike (resp. timelike) Jordan Ivanov-Petrova.

Proof. As $g_{1}=\alpha g_{2}$, one has $W_{g_{1}}=\alpha W_{g_{2}}$; the Weyl conformal curvature tensor simply rescales. Let $x \in T_{P} M$ be a $g_{2}$ spacelike or timelike unit vector. Let

$$
\tilde{x}:=\frac{1}{\sqrt{\alpha(P)}} x
$$

be the corresponding $g_{1}$ spacelike or timelike unit vector. Similarly, if $\left\{e_{1}, e_{2}\right\}$ is an oriented $g_{2}$ orthonormal basis for $\pi$, then

$$
\left\{\frac{1}{\sqrt{\alpha}} e_{1}, \frac{1}{\sqrt{\alpha}} e_{2}\right\}
$$

is the corresponding oriented $g_{1}$ orthonormal basis for $\pi$. We then have

$$
J_{W_{g_{1}}}(\tilde{x})=\frac{1}{\alpha(P)} J_{W_{g_{2}}}(x) \quad \text { and } \quad W_{g_{1}}(\pi)=\frac{1}{\alpha(P)} W_{g_{2}}(\pi)
$$

The Lemma now follows as the Jordan normal forms are simply rescaled.

Theorem 1.1 shows that the notions we are studying are well defined in conformal geometry and justifies the notation we have employed. Here is a brief guide to the remainder of the paper. In Section 2, we will present some results concerning conformally spacelike and timelike Jordan Osserman manifolds. In Section 3, we will present some results concerning conformally spacelike and timelike Jordan Ivanov-Petrova manifolds. We conclude in Section 4 with some examples.

2. Conformally Jordan Osserman manifolds. We begin with the following observation: 
THEOREM 2.1. If $(M, g)$ is Einstein, then $(M, g)$ is conformally spacelike (resp. timelike) Jordan Osserman if and only if $(M, g)$ is pointwise spacelike (resp. timelike) Jordan Osserman.

Proof. If $(M, g)$ is Einstein, then Equation (1.b) implies

$$
g\left(J_{W}(x) y, z\right)=g\left(J_{R}(x) y, z\right)+\lambda\{g(y, z) g(x, x)-g(y, x) g(z, x)\}
$$

where $\lambda$ is a suitably chosen constant. Thus

$$
J_{W}(x) y= \begin{cases}0 & \text { if } y=x, \\ \left\{J_{R}(x)+\lambda g(x, x) \operatorname{Id}\right\} y & \text { if } y \perp x .\end{cases}
$$

Thus apart from the trivial eigenvalue 0 , the Jordan normal form of $J_{W}(x)$ and $J_{R}(x)$ are simply shifted by adding a scalar multiple of the identity if $x$ is not a null vector. Theorem 2.1 is now immediate.

The classification is complete in certain settings:

TheOrem 2.2. Assume either that $(M, g)$ is an odd dimensional Riemannian manifold or that $(M, g)$ is a Lorentzian manifold. Then $(M, g)$ is conformally spacelike Jordan Osserman if and only if $(M, g)$ is conformally flat.

Proof. We say $\mathcal{V}=(V, g, A)$ has constant sectional curvature $\lambda$ if $A=\lambda A_{\text {Id }}$, i.e.

$$
A(x, y, z, w)=\lambda\{g(x, w) g(y, z)-g(x, z) g(y, w)\} .
$$

If $\mathcal{V}$ is Riemannian spacelike Jordan Osserman and if the dimension $m$ is odd, then work of Chi [3] shows that $\mathcal{V}$ has constant sectional curvature. If $\mathcal{V}$ is Lorentzian and spacelike Jordan Osserman, then results of Blažić, Bokan and Gilkey [1] and of García-Río, Kupeli and Vázquez-Abal [5] shows that $\mathcal{V}$ has constant sectional curvature.

If $A$ has constant sectional curvature $\lambda$ and if $x$ is not null, then Equation (1.c) shows

$$
J_{A}(x)(y)= \begin{cases}0 & \text { if } y=x, \\ \lambda g(x, x) y & \text { if } y \perp x .\end{cases}
$$

Consequently $\operatorname{Tr}\left(J_{A}(x)\right)=(m-1) \lambda g(x, x)$. Therefore, if $A \in \mathcal{W}(V, g)$, then necessarily $\lambda=0$ by Equation (1.d). Theorem 2.2 now follows by applying these observations to $\mathcal{V}:=\left(T_{P} M, g_{P}, W_{P}\right)$.

One says that a manifold is spacelike (resp. timelike) Osserman if the eigenvalues of the Jacobi operator are constant on the pseudo-sphere $S^{+}\left(T_{P} M\right)$ (resp. $S^{-}\left(T_{P} M\right)$ ) for any point $P \in M$. Theorem 2.2 extends to show that any Lorentzian manifold $(M, g)$ which is spacelike (resp. timelike) Osserman is conformally flat.

Any local rank 1 Riemannian symmetric space is necessarily conformally Osserman since the group of local isometries acts transitively on the unit sphere bundle. We conjecture that the converse holds; this is the analogue of the Osserman conjecture in this setting:

Conjecture 2.3. A connected Riemannian manifold $(M, g)$ is conformally Osserman if and only if $(M, g)$ is locally conformally equivalent to a rank 1 symmetric space.

We shall see in Section 4 that this conjecture fails in the higher signature setting. 
3. Conformally Jordan Ivanov-Petrova manifolds. The classification is almost complete in the Riemannian setting:

TheOrem 3.1. Let $(M, g)$ be a conformally spacelike Jordan Ivanov-Petrova Riemannian manifold of dimension $m \neq 3,7$. Then $(M, g)$ is conformally flat.

Proof. Suppose first $m \geq 5$ and $m \neq 7$. We apply results of [8,11] to see that any Riemannian Ivanov-Petrova algebraic curvature tensor in these dimensions has rank 2. Such tensors are classified. Let $P \in M$. There exists a self-adjoint isometry $\phi_{P}$ of $T_{P} M$ with $\phi_{P}^{2}=$ Id so that $W_{P}=\lambda R_{\phi_{P}}$ where $R_{\phi_{P}}$ is given by Equation (1.a). We may then use Equation (1.c) to see:

$$
J_{W}(x) y=\lambda g_{P}\left(\phi_{P} x, x\right) \phi_{P} y \quad \text { if } \quad y \perp \phi_{P} x .
$$

Decompose $T_{P} M=T_{P}^{+} M \oplus T_{P}^{-} M$ into the \pm 1 eigenspaces of $\phi_{P}$. Let $e^{ \pm}$be unit vectors in $T_{P}^{ \pm} M$. Set $a^{ \pm}:=\operatorname{dim} T_{P}^{ \pm} M$. Then Equation (3.a) implies that

$$
\operatorname{Tr}\left\{J_{W}\left(e^{+}\right)\right\}=\lambda\left(a^{+}-1-a^{-}\right) \quad \text { and } \operatorname{Tr}\left\{J_{W}\left(e^{-}\right)\right\}=\lambda\left(a^{-}-1-a^{+}\right) .
$$

If $\phi= \pm \mathrm{Id}$, then $W_{P}$ has constant sectional curvature and the argument given to establish Theorem 2.2 shows $W_{P}=0$. Thus we may assume that $a^{+} \geq 1$ and $a^{-} \geq 1$. By Equation (1.d), $\operatorname{Tr}\left\{J_{W}(x)\right\}=0$ for any $x$. Thus we have

$$
\left(a^{+}-a^{-}-1\right) \lambda=0 \text { and }\left(a^{-}-a^{+}-1\right) \lambda=0 .
$$

Adding these two equations implies $-2 \lambda=0$ and hence $W_{P}=0$. This establishes the Lemma except when $m=4$.

We complete the proof of the Lemma by dealing with the exceptional case $m=4$. We follow the discussion in Ivanov-Petrova [17] to see that either $W$ has the form given in Equation (3.a), in which case the argument given above shows $W_{P}=0$, or that there exists an orthonormal basis $\left\{e_{1}, e_{2}, e_{3}, e_{4}\right\}$ for $T_{P} M$ so that the non-zero components of $W$ are given by:

$$
\begin{array}{clll}
W_{1212}=a_{1}, & W_{1234}=a_{2}, & W_{1313}=a_{2}, & W_{1324}=-a_{1}, \\
W_{1414}=a_{2}, & W_{1423}=a_{1}, & W_{2323}=a_{2}, & W_{2314}=a_{1}, \\
W_{2424}=a_{2}, & W_{2413}=-a_{1}, & W_{3434}=a_{1}, & W_{3412}=a_{2} .
\end{array}
$$

where $a_{2}+2 a_{1}=0$. Since $\rho_{W}\left(e_{1}, e_{1}\right)=-2 a_{2}-a_{1}=0$ by Equation (1.d), we conclude $a_{1}=a_{2}=0$, which once again implies $W_{P}=0$.

There are analogous results in the higher signature setting, although with slightly more restrictive hypotheses.

TheOREM 3.2. Let $(M, g)$ be a connected pseudo-Riemannian manifold of signature $(p, q)$ which is conformally spacelike Ivanov-Petrova. Assume that $q \geq 11$, that $p \leq \frac{q-6}{4}$, and that $\{q, q+1, \ldots, q+p\}$ does not contain a power of 2 . Then either $W(\pi)$ is nilpotent for every spacelike 2 plane or $(M, g)$ is conformally flat.

Proof. Results of $[15,16,21]$ show that there exists a normalizing constant $\lambda$ so that $W_{P}=\lambda W_{\phi}$ where $W_{\phi}$ is given by Equation (1.a) where one of the following conditions holds: 
1. $\phi^{2}=\mathrm{Id}$ and $\phi$ is a self-adjoint isometry of $T_{P} M$.

2. $\phi^{2}=-\mathrm{Id}$ and $\phi$ is a self-adjoint para-isometry of $T_{P} M$.

3. $\phi^{2}=0$.

If $\phi^{2}=0$, then $W(\pi)$ is always nilpotent. We complete the proof by showing that either (1) or (2) imply $\lambda=0$.

Suppose $\phi$ is a self-adjoint isometry of $T_{P} M$ with $\phi^{2}=\mathrm{Id}$. As in the proof of Theorem 3.1 , we decompose $T_{P} M=T_{P}^{+} M \oplus T_{P}^{-} M$ into the \pm 1 eigenspaces of $\phi$. Again, we set $a^{ \pm}=\operatorname{dim} T_{P}^{ \pm}$were we may suppose $a^{+} \geq 1$ and $a^{-} \geq 1$. These eigenspaces are orthogonal with respect to the metric $g_{P}$ and thus the restriction of the metric to each eigenspace is non-degenerate. Thus we may choose vectors $e^{ \pm} \in T_{P}^{ \pm} M$ so $g_{P}\left(e^{ \pm}, e^{ \pm}\right)=\varepsilon^{ \pm} \neq 0$. Equation (3.b) then extends to become

$$
\operatorname{Tr}\left\{J_{W}\left(e^{+}\right)\right\}=\varepsilon^{+} \lambda\left(a^{+}-1-a^{-}\right) \text {and } \operatorname{Tr}\left\{J_{W}\left(e^{-}\right)\right\}=\varepsilon^{-} \lambda\left(a^{-}-1-a^{+}\right) .
$$

We argue as in the proof of Theorem 3.1 to see that this implies $\lambda=0$.

If $\phi$ is a para-isometry, we complexify. Replacing $\phi$ by $\tilde{\phi}:=\sqrt{-1} \phi$ and applying the argument given above to the $g$ self-adjoint (complex) isometry $\tilde{\phi}$ to see that $\sqrt{-1} \lambda=0$ and thus, again, $W_{P}=0$.

This result, together with the examples in the subsequent section, motivates the following:

Conjecture 3.3. Let $(M, g)$ be a conformally spacelike Ivanov-Petrova manifold. If $(M, g)$ is not conformally flat, then $W(\pi)$ is nilpotent for any oriented spacelike 2-plane $\pi$.

4. Examples. Theorem 2.2 shows that Conjecture 2.3 holds if $m$ is odd. The situation is considerably more complicated in the higher signature setting. The following family of manifolds [10] is useful in this setting. It also shows there are conformally spacelike Jordan Ivanov-Petrova manifolds which are not conformally flat. Let $p \geq 2$. Introduce coordinates $\left(x_{1}, \ldots, x_{p}, y_{1}, \ldots, y_{p}\right)$ on $\mathbb{R}^{2 p}$ and let $f=f\left(x_{1}, \ldots, x_{p}\right)$ be a smooth function on $\mathbb{R}^{2 p}$. Define a neutral signature metric $g_{f}$ on $\mathbb{R}^{2 p}$ by setting

$$
g_{f}\left(\partial_{i}^{x}, \partial_{j}^{x}\right):=\partial_{i}^{x} f \cdot \partial_{j}^{x} f, \quad g_{f}\left(\partial_{i}^{x}, \partial_{j}^{y}\right)=g_{f}\left(\partial_{j}^{y}, \partial_{i}^{x}\right)=\delta_{i j}, \quad g_{f}\left(\partial_{i}^{y}, \partial_{j}^{y}\right)=0 .
$$

Let $H=\left(H_{i j}\right) \in M_{p}(\mathbb{R})$ be the Hessian where $H_{i j}=\partial_{i}^{x} \partial_{j}^{x}$.

Theorem 4.1. Let $\left(M, g_{f}\right)$ be as defined above. Assume that $p \geq 3$.

1. Assume that $H$ is definite. If $x$ is not null, then $J_{W}(x)$ has rank $p-1$ and $J_{W}(x)^{2}=0$. Thus $\left(M, g_{f}\right)$ is conformally spacelike and timelike Jordan Osserman.

2. If $H$ is indefinite, then $\left(M, g_{f}\right)$ is neither conformally spacelike Jordan Osserman nor conformally timelike Jordan Osserman.

3. Assume that $H$ is non-degenerate. If $\pi$ is an oriented spacelike or timelike 2 plane, then $\operatorname{Rank}(W(\pi))=2$ and $W(\pi)^{2}=0$. Thus $\left(M, g_{f}\right)$ is conformally spacelike and timelike Jordan Ivanov-Petrova.

Proof. We showed in [10] that $\left(M, g_{f}\right)$ was Ricci flat. Consequently, $W=R$. The assertions of Theorem 4.1 now follow from the corresponding assertions for $J_{R}(x)$ and for $R(\pi)$ which were established in [10]. 
The manifolds of Theorem 4.1 have a Jacobi operator and skew-symmetric curvature operator which are nilpotent of order 2. There are also manifolds where the Jacobi operator and skew-symmetric curvature operator are nilpotent of order 3 . Let $\left(u_{1}, \ldots, u_{s}, t_{1}, \ldots, t_{s}, w_{1}, \ldots, w_{s}\right)$ be coordinates on $\mathbb{R}^{3 s}$ for $s \geq 2$. Let $f_{i}(x)$ be smooth functions on $\mathbb{R}$ and set $F\left(u_{1}, \ldots, u_{s}\right):=f_{1}\left(u_{1}\right)+\ldots+f_{s}\left(u_{s}\right)$. Define a metric of signature $(2 s, s)$ on $M_{F}:=\mathbb{R}^{3 s}$ by setting

$$
\begin{array}{ll}
g_{F}\left(\partial_{i}^{u}, \partial_{j}^{u}\right)=-2 \delta_{i j} F(u)-2 \delta_{i j} \sum_{k} u_{k} t_{k}, & g_{F}\left(\partial_{i}^{u}, \partial_{j}^{v}\right)=g_{F}\left(\partial_{j}^{v}, \partial_{i}^{u}\right)=\delta_{i j}, \\
g_{F}\left(\partial_{i}^{u}, \partial_{j}^{t}\right)=g_{F}\left(\partial_{j}^{t}, \partial_{i}^{u}\right)=0, & g_{F}\left(\partial_{i}^{t}, \partial_{j}^{t}\right)=-\delta_{i j}, \\
g_{F}\left(\partial_{i}^{t}, \partial_{j}^{v}\right)=g_{F}\left(\partial_{j}^{v}, \partial_{i}^{t}\right)=0, & g_{F}\left(\partial_{i}^{v}, \partial_{j}^{v}\right)=0 .
\end{array}
$$

TheOREM 4.2. Let $\left(M_{F}, g_{F}\right)$ be as defined above where $s \geq 2$.

1. Let $x$ be spacelike. Then $J_{W}(x)$ has rank $2 s-2, J_{W}(x)^{2}$ has rank $s-1$, and $J_{W}(x)^{3}=0$. Consequently $\left(M_{F}, g_{F}\right)$ is conformally spacelike Jordan Osserman. However, $\left(M_{F}, g_{F}\right)$ is not conformally timelike Jordan Osserman.

2. Let $\pi$ be an oriented spacelike 2-plane. Then $W(\pi)$ has rank $4, W(\pi)^{2}$ has rank 2 , and $W(\pi)^{3}=0$. Consequently $\left(M_{F}, g_{F}\right)$ is conformally spacelike Jordan Ivanov-Petrova. However, $\left(M_{F}, g_{F}\right)$ is not conformally timelike Jordan Ivanov-Petrova.

Proof. We showed in [12] that $\left(M_{F}, g_{F}\right)$ is Ricci flat. Consequently, $R=W$. The assertions of the Theorem now follow from the corresponding assertions for $J_{R}$ which were established in [12] and for $W(\pi)$ which were established in [14].

Acknowledgments. Research of N. Blažić partially supported by the DAAD (Germany) and MNTS Project \#1854 (Srbija). Research of P. Gilkey partially supported by the MPI (Leipzig). Research of S. Nikčević partially supported by the DAAD (Germany) and MMTS \#1646 (Srbija). Research of U. Simon partially supported by DFG-Si163/7-2. All authors were partially supported by VolkswagenStiftung. The authors wish to express their thanks to the Technische Universität Berlin where much of the research reported here was conducted.

\section{References}

[1] N. Blažić, N. Bokan, and P. Gilkey, A note on Osserman Lorentzian manifolds, Bull. London Math. Soc. 29 (1997), 227-230.

[2] N. Blažić, N. Bokan, and Z. Rakić, Osserman pseudo-Riemannian manifolds of signature $(2,2)$, Aust. Math. Soc. 71 (2001), 367-395.

[3] Q.-S. Chi, A curvature characterization of certain locally rank-one symmetric spaces, J. Differential Geom. 28 (1988), 187-202.

[4] B. Fiedler, Determination of the structure of algebraic curvature tensors by means of Young symmetrizers, Seminaire Lotharingien de Combinatoire B48d (2003). 20 pp. Electronically published: http://www.mat.univie.ac.at/ slc/; see also math.CO/0212278.

[5] E. García-Río, D. Kupeli, and M. E. Vázquez-Abal, On a problem of Osserman in Lorentzian geometry, Differential Geom. Appl. 7 (1997), 85-100. 
[6] E. García-Río, D. Kupeli, and R. Vázquez-Lorenzo, Osserman Manifolds in SemiRiemannian Geometry, Lecture Notes in Mathematics 1777, Springer-Verlag, Berlin, 2002.

[7] E. García-Rió, M. E. Vázquez-Abal, and R. Vázquez-Lorenzo, Nonsymmetric Osserman pseudo-Riemannian manifolds, Proc. Amer. Math. Soc. 126 (1998), 2771-2778.

[8] P. Gilkey, Riemannian manifolds whose skew-symmetric curvature operator has constant eigenvalues II, in: Differential Geometry and Applications (Brno, 1998), Masaryk Univ., Brno, 1999, 73-87.

[9] P. Gilkey, Geometric Properties of Natural Operators Defined by the Riemann Curvature Tensor, World Scientific, River Edge, NJ, 2001.

[10] P. Gilkey, R. Ivanova, and T. Zhang, Szabó Osserman IP pseudo-Riemannian manifolds, Publ. Math. Debrecen 62 (2003), 387-401.

[11] P. Gilkey, J. V. Leahy, and H. Sadofsky, Riemannian manifolds whose skew-symmetric curvature operator has constant eigenvalues, Indiana Univ. Math. J. 48 (1999), 615-634.

[12] P. Gilkey and S. Nikčević, Curvature homogeneous spacelike Jordan Osserman pseudoRiemannian manifolds, Classical Quantum Gravity 21 (2004), 497-507.

[13] P. Gilkey and S. Nikčević, Nilpotent spacelike Jordan Osserman pseudo-Riemannian manifolds, Proc. Winter School "Geometry and Physics", Srni (2003), Rendiconti Circolo Matematico Palermo, Ser. II, Suppl. 72 (2004), 99-105.

[14] P. Gilkey, S. Nikčević, and V. Videv, Manifolds which are Ivanov-Petrova or $k$-Stanilov, J. Geom. 80 (2004), 82-94.

[15] P. Gilkey and T. Zhang, Algebraic curvature tensors whose skew-symmetric curvature operator has constant rank 2, Periodica Mathematica Hungarica 44 (2002), 7-26.

[16] P. Gilkey and T. Zhang, Algebraic curvature tensors for indefinite metrics whose skewsymmetric curvature operator has constant Jordan normal form, Houston J. Math. 28 (2002), 311-328.

[17] S. Ivanov and I. Petrova, Riemannian manifolds in which the skew-symmetric curvature operator has pointwise constant eigenvalues, Geom. Dedicata 70 (1998), 269-282.

[18] Y. Nikolayevsky, Two theorems on Osserman manifolds, Differential Geom. Appl. 18 (2003), 239-253.

[19] Y. Nikolayevsky, Osserman manifolds of dimension 8, Manuscr. Math. 115 (2004), 31-53.

[20] Y. Nikolayevsky, Osserman Conjecture in dimension $n \neq 8,16$, Math. Annalen 331 (2005), 505-522.

[21] I. Stavrov, Spectral geometry of the Riemann curvature tensor, Ph.D. Thesis, University of Oregon, 2003. 